\title{
Physics-based prognostic modelling of filter clogging phenomena
}

\author{
Omer F. Eker ${ }^{\mathrm{a}, \mathrm{c}}$, FatihCamci ${ }^{\mathrm{b}}$, Ian K. Jennions ${ }^{\mathrm{a}}$ \\ ${ }^{a}$ IVHM Centre, Cranfield University,MK43 OAL, UK \\ i.jennions@cranfield.ac.uk \\ ${ }^{\mathrm{b}}$ Industrial Engineering, Antalya International University, Turkey \\ fatih.camci@antalya.edu.tr \\ ${ }^{\mathrm{c}}$ Artesis Technology Systems, GOSB Technopark, Turkey \\ omer.eker@atrtesis.com
}

\begin{abstract}
In industry,contaminant filtration is a common process to achieve a desired level of purification, since contaminants in liquids such as fuel may lead to performance drop and rapid wear propagation. Generally, clogging of filter phenomena is the primary failure mode leading to the replacement or cleansing of filter. Cascading failures and weak performance of the systemare the unfortunate outcomesdue to a clogged filter. Even though filtration and clogging phenomena and their effects of several observable parameters have been studied for quite some time in the literature, progression of clogging and its use for prognostics purposes have not been addressed yet. In this work, a physics based clogging progression model is presented. The proposed model that bases on a well-known pressure drop equation is able to model three phases of the clogging phenomena, last of which has not been modelled in the literature yet. In addition, the presented model is integrated with particle filters to predict the future clogging levels and to estimate the remaining useful life of fuel filters. The presented model has been implemented on the data collected from an experimental rig in the lab environment. In the rig, pressure drop across the filter, flow rate, and filter mesh images are recorded throughout the accelerated degradation experiments. The presented physics based model has been applied to the data obtained from the rig. The remaining useful livesof the filters used in the experimental rig have been reported in the paper.The results show that the presented methodology provides significantly accurate and precise prognostic results.
\end{abstract}

Keywords: Prognostics and health management, Physics-based modelling, Filter clogging modelling

\section{Introduction}

Filtration is basically described as a unit operation that is separation of suspended particles from the fluid, utilising a filtering medium, where only the fluid can pass [1]. Filtration is important in many engineering systems in automotive, chemical, food, petroleum, pharmaceuticals, metal production, and reactors applications [2].For example, filtration and separation equipment plays a substantial portion (15\%) in production of transport equipment manufacturing. Modern commercial vehicles and automobiles have numerous types of filters including fuel, lubricant, and intake air [3].Fuel filters clean dirt and other contaminants in the fuel system such as sulphates, polymers, paint chips, dust, and rust particulate which are released from the fuel tank due to moisture as well as other numerous types of dirt have been uplifted via supply tanker [4] and[5]. Consequences such as engine and pump performance degradation due to increased abrasion and inefficient burning in the engine are the main motivators for fuel filtration leading to a purified fuel. However, fuel filtering associates with some complications such as clogging of filter. This paper deals with filter clogging phenomena and physical modelling of filter clogging progression for prognostics purposes.

The aim of the filtration systems is to keep the rest of the system running smoothly; moreover, they play a vital role in maintaining the process in normal operating state. System flow rate and engine performance declines once a fuel filter is clogged where it does not function well in its desired operation ranges. Jones[5] reports that filter clogging indication due to fuel contamination may result in an aircraft having to return to the ground or divert for further fuel filter inspection or replacement. Fuel filter bypass indicator alarms the pilot and the crew to take an action when the pressure drop level reaches a predefined threshold (e.g. 11.5 \pm 1 PSI for GE CFM53-3 engine). A potential catastrophic failure such as engine shutdown may occur when both engines are exposed to unfiltered fuel due to the bypass operation. Even though clogged filter may enhance the filtering process, it is not acceptable to use clogged filters due to other system related factors such as performance, efficiency, etc.

*Corresponding author. Tel: +90-242 245 0342; E-mail: fatih.camci@antalya.edu.tr 
In traditional maintenance approach, fuel filters are replaced or cleansed on a regular basis.For example, Boeing 777 fuel filter are replaced at every two thousand flight hours[5]. This approach may lead to usage of clogged filter before completion of the predefined period for replacement or early replacement of the filter even though it may be used for extra some time. Increasing the replacement period increases probability of failures due to clogged filter, whereas reducing it increases the waste of resources such as filter, replacement labour related costs etc. Prognostics and Health Management (PHM) is relatively new approach aiming to balance the cost of early replacement/maintenance and cost of increased failure probability due to usage of the components until their end of lives through continuous monitoring, diagnostics and prognostics. Continuous monitoring is achieved using sensors embedded into the close proximity to the equipment aiming to quantify the symptoms occurred due to the failure. Diagnostics is the process of detection of an existing or developing failure, identification of its severity, and localisation of the failure. Prognostics is the process of identification of the time that the failure will make the system not to perform its intended function. In other words, prognostics aims to estimate the remaining useful life (RUL) of the system before the failure. Prognostics is thought to be the future of maintenance management in industrial systems with increasing complexity, maintenance and failure costs. Prognostics of filter clogging phenomena is the main focus of this paper.

Prognostics involves two steps. The first step is the identification of the current health state, whereas the second step is the forecasting the progression of the health state in the future time. Physics based (or Model-Based)and data driven methods are the types of prognostics methods. Physics based models aim to formulate the physical phenomena occurring during the failure progression, whereas data driven approach models the data collected sensors during the failure progression without considering physical meanings. Physics-based models are generally expected to be the first choice over other methods as they are known for their accuracy and precision when a good representative of a mathematical model is driven or available. They also require less data compared to a data-driven approach and suitable for creation in the design phase of equipment. However they are costly to implement and difficult to create an accurate model specifically for complex systems. This paper focuses on the physics based prognosticsfor filter clogging phenomena. Even though filter clogging has been extensively studied in the literature, existing physics based models in the literature are not able to fully represent the clogging phenomena. In addition, progression of clogging has not been addressed in these models, which is the major step for prognostics. This research aims to develop a prognostics methodology by addressing these problems through a new physics based model.

This article is organised as follows: Section 2 gives literature review; Section 3 presentsa new physics based filter clogging prognostic model. Section 4 presents the experimental rig design, data collection and prognostics results. Section 5 concludes the paper as well as laying out the future directions.

\section{Literature review}

Solid-liquid filtration processes can be classified into three categories: 1. Deep-bed filtration, 2. Cross-flow filtration, and 3. Cake filtration. Deep-bed filtration can be performed using depth-filters. Depth filters retain the particulate through a porous packed bed. Sand filters are the common examples of depth filtration. In cross-flow filtration mechanism, slurry flows parallel to the filter medium where only clean liquid can pass to the other side, leaving the particulate inside the filter. In cake filtration, solid particles in a suspension, flowing through a filter media, are retained on a filter medium, building up an increasing thicker cake as shown inFig.1. As the cake layer becomes thicker, the cake structure becomes the main filtration component, leading to an excessively flow resistance in the final stages of filtration process. Eventually the flow rate drops to a certain level due to filter plugging, creating a need for cleansing or replacement of the filter.Ni et al. [6] reports that the cake filtration process takes part in many industries including; mineral, chemical, pharmaceutical, food, and petroleum. This paper focuses on the cake filtration category.

In the literature, the cake filtrationis conducted in two different operation types: Constant flow rate and constant pressure filtration. In the former, the filter is fairly clean and constant flow rate is achieved during the filtration process. The fluid flow rate is constant in this phase, whereas pressure drop across the filtration medium increases as the cake builds up. Pressure drop increase may exhibit a stochastic logarithmic or exponential character. In most cases cake becomes 


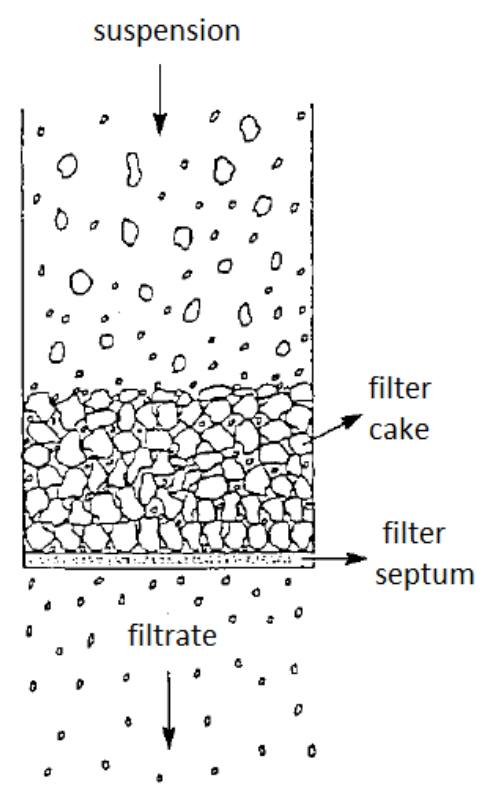

Fig.1: Schematic representation of cake build-up on filter medium [10]

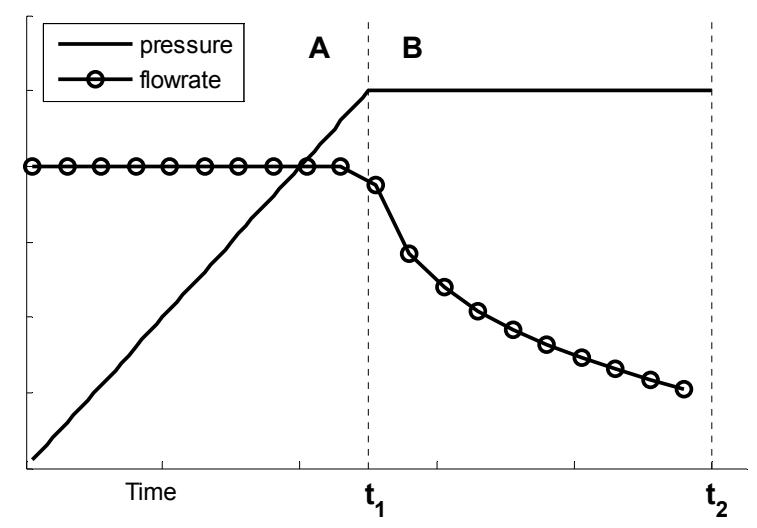

Fig.2: Constant rate vs. constant pressure filtration

compressed and more compact as the pressure increases, leading to higher cake resistance, which may lead to a constant pressure filtration regime [7], [8] and [9]. In the latter type of operation, constant pressure drop is achieved, where the flow rate of the system declines as the cake builds up.Fig.2depicts the flow rate and pressure drop behaviours in both phases. Time until $t_{1}$ represents the constant flow rate phase, whereas the time after $t_{1}$ represent constant pressure drop phase.

Filter clogging phenomena is highly related to the fluid flow rate and pressure difference before and after the filter, since clogging leads to reduced fluid flowand/or increased pressure drop. Researches have been attracted to model the fluid flow through a porous media since early 1900s. One of the earliest models for this type of flow is proposed by Forchheimer[11]. His simple model associates pressure drop to fluid flow, given in Eq. (1) where ' $\Delta \mathrm{p}$ ' is the pressure drop across the porous medium, ' $V_{s}$ ' is the flow velocity;parameters ' $a$ ' and ' $b$ ' in the equation are the constants characterising the filter medium.This model has served a basis for several complex models in the future (e.g. Kozeny-Carman, Ergun, and Endo equations).

$$
\Delta p=a V_{s}+b V_{s}^{2}
$$

Darcy's Law is another early model which has been used for calculating the permeability of a filter septum [12]. Darcy described the volumetric flow rate ' $Q$ ' of a system as a function of pressure drop ' $\Delta \mathrm{p}$ ', permeability ' $\mathrm{K}$ ', cross sectional area to flow 'A', viscosity ' $\mu$ ' of the fluid, and the thickness ' $L$ ' of the porous medium (e.g. depth of a deep bed filter) as shown in Eq. (2). 


$$
Q=\frac{K A}{\mu L} \Delta p
$$

Kozeny-Carman [13] and Ergun [14] equations are two of the commonly used formulations applied in fluid dynamics to model the pressure drop of a fluid flowing through a porous medium (e.g. packed bed, filter mesh). Tien and Ramarao[11]questionedKozeny-Carman equations especially when 'porosity' (i.e. void fraction of the filtration medium) modelling of compressible and randomly packed filter cakes are used in gas-solid separation processes. They claimed that Kozeny-Carman is more appropriate when it is used only for pressure drop-flow rate correlations.

Endo et al. [9] reports that Kozeny-Carman or the extended version (e.g. Ergun equation) can only be applied to the particles with a narrow size distribution. They developed a novel pressure drop model incorporating the particle size distribution and particle shape factor.Conventional cake filtration theory has the capability of estimating the cake thickness, cake resistance, porosity, and pressure drop in the system. Tien and Bai [8] discussed a more accurate procedure of applying the conventional cake filtration theory. They reported that the cake thickness and compressibility of the cake have the highest influence on pressure drop across the filter.

Several methods have been implemented to measure the cake thickness depending on the filter geometry including ultrasonic, electrical conductivity techniques, nuclear magnetic resonance micro-imaging, optical observation, and cathetometer measuring [15]. Ni et al. [6] have modelled cake formation and pressure drop of a filtration mechanism in particle level (i.e. micro level) where majority of the studies in literature are conducted in macro level. They simulated the cake filtration process in both constant pressure and constant rate stages. Liu et al. [16] implemented pressure drop modelling on the impact of membrane diesel particulate filter based on Endo's extended version of Kozeny-Carman equations. In their model, they correlated the pressure drop across a type of membrane filters to diesel exhaust gas particulate retention parameters.

Several studies on applicationsof filtration process on different platforms have been reported in the literature. Park[17] has investigated F-5F aircraft engine failure caused by erosion-corrosion of a fuel manifold, claiming the engine failures are caused by sudden pressure drop due to particles (e.g. mostly steel and iron) from the welding beads of fuel manifold. Internal welding beads are corroded and metal particles spread out which makes the fuel pump failed. The results are obtained by using energy-dispersive X-ray spectroscopy (EDX) analysis of related surfaces. A comprehensive investigation of unmanned aerial vehicle (UAV) fuel systems has been conducted in IVHM Centre, Cranfield University, UK [18] and[19]. Several failure scenarios including clogged filter and faulty gear pump are investigated; particularly diagnostics-based studies are conducted.

Clogging process of different types of filtration mechanisms has been studied in the literature.Roussel et al.[20] presented a particle level filtration case study; stating that the general clogging process can be considered as a function of: Ratio of particle to mesh pore size, solid fraction, and the number of grains arriving at each mesh hole during one test. The group conducted several clogging experiments and optimised the clogging parameters in their model. Their studies may help to model the first regime of cake filtration clogging process.Sappok et al.[21] worked on the effects of ash accumulation in diesel particulate filters (DPF). They presented detailed measurement results with formulated lubricants, correlating ash properties to individual lubricant additives and their effects on filter pressure build-up.Pontikakis et al.[22] developed a mathematical model for dynamic behaviour of filtering process for ceramic foam filters. The model is capable of estimation of the filtration efficiency, accumulation of particle mass in the filter, and the pressure drop throughout the filter.Roychoudhury et al. [23] presented a diagnostic and prognostic solution for water recycling system for next generation spacecrafts. They simulated several failure scenarios including clogging of membranes and filters.Baraldi et al.[24] and[25] developed a similarity-based and Gaussian process regression (GPR) prognostic approach to estimate the remaining useful life (RUL) of sea water filters.Saarela et al. [26] presented a nuclear research reactor air filter pressure drop modelling scheme which utilises gamma processes. These methodologies do base on a physics based clogging progression. This paper also aims to develop a physics based clogging progression model that can be used for prognostics purposes.

Even though there exist many studies in filter clogging focusing on physical modelling of clogging phenomena; there is a lack of filter clogging progression prediction model that can be used in prognostics ofan engineering system. This paper aims to fill this gap by presenting a physics based model and using this model for remaining useful life estimation of a fuel filter. In addition, the filter clogging models presented in the literature has inability to model the whole life of the filter in the clogging phenomena. In other words, the existing methods are able to model the filter clogging until a point in the clogging process. The behaviour after that point cannot be captured by the existing methods. Thus, the main driver of this paper is to fill these gaps by creating a model that is able to fully model the clogging phenomenathat can be used for prognostics purposes. 


\section{Methodology}

This chapter includes two subsections. The first one presents the physical model representing all three phases of the clogging phenomena. The latter presents the integrationof this model with the Particle Filters for prognostics purposes.

\subsection{Physical model for filter clogging phenomena}

The severity of the filter clogging is the main parameter in identification of the replacement time for the filter. The direct measure of the severity may not be possible during the usage of the system through continuous monitoring in PHM. The aim of the physical model is to calculate the severity of the filter clogging using the measurable parameters during the system usage. Pressure drop across the filter, volumetric flow rate, cake thickness, and porosity are the main dynamic parameters revealing the clogging severity of the filter. It may be feasible and easy to measure some of these parameters. If direct measure is not possible for some of them, some other measures may be used to drive them. Ergun equation formulates the relationship between pressure drop and the other clogging parameters as given in Eq. (3). The void function of porosity (i.e. ' $v(\epsilon)$ ') has other complex forms for different types of applications [16]. A version of $v(\epsilon)$ used in Ergun and Kozeny-Carman equations is given in Eq. (4). The Ergun equation with the given $v(\epsilon)$ formula is re-written as in (5).

$$
\begin{aligned}
& \Delta P=\frac{A V_{S} \mu(1-\epsilon) v(\epsilon) L}{D_{p}^{2} \epsilon^{2}}+\frac{B(1-\epsilon) \rho V_{s}^{2} L}{\epsilon^{3} D_{p}} \\
& v(\epsilon)=\frac{10(1-\epsilon)}{\epsilon} \\
& \Delta P=\frac{10 A V_{S} \mu(1-\epsilon)^{2} L}{D_{p}^{2} \epsilon^{3}}+\frac{B(1-\epsilon) \rho V_{S}^{2} L}{\epsilon^{3} D_{p}}
\end{aligned}
$$

Where $\Delta \mathrm{P}$ is the: pressure drop (upstream pressure-downstream pressure), $\mathrm{v}(\epsilon)$ is the void function of porosity, $\mathrm{L}$ is the total height of the bed (e.g. cake thickness), $\epsilon$ is the porosity of the bed (or cake), $V_{s}$ is the superficial (empty-tower) velocity, $\mu$ is the viscosity of the fluid, $D_{p}$ is the diameter of the spherical particle, $\rho$ is the liquid density, and $A, B$ are the constants.

According to the equation; viscosity and velocity of fluid and thickness of cake are the parameters which raise the pressure drop across cake when they increase, in contrast to particle diameter and porosity parameters. The Ergun equation is a detailed version of the renowned Kozeny-Carman equation. Tien and Ramarao[11] claimed that the Ergun equation is the most commonly used model which is capable of describing the pressure drop and flow rate correlation. The first term in the Ergun equation represents viscous effect whereas the second term associates with the inertial effect which is not taken into account in Kozeny-Carman model.

This study proposes a modified version of Ergun equation that incorporates effective filtration area in the filter. Effective filtration area reduces significantly after the clogging reaches to a severity level, where the third phase of the filter clogging starts. In the third phase in filter clogging, the cake height is restricted to grow by the filter container creating other forces affecting the measured parameters. These effects have not been modelled in the literature yet. Therefore, a new parameter is defined for measuring the effective filtration area, called effective filtration area rate $(a)$. Effective filtration area rate is defined as the rate of the filtration area of the particle deposit cake inside the filter chamber where fluid can pass at a time to its initial value in no clogging case (when the filter is clean). The parameter ' $a$ ' representing the effective filtration area rate assists modelling the third phase of the filtration process. The modified version of Ergun equation is given below:

$$
\Delta P=\frac{10 A V_{S} \mu(1-\epsilon)^{2} L}{D_{p}^{2} \epsilon^{3} a}+\frac{B(1-\epsilon) \rho V_{S}^{2} L}{\epsilon^{3} D_{p} a}
$$

The parameter ' $a$ ' is a dynamic variable, driven by the sphere packing simulation modelling. However this rate reduces dramatically when the deposited particles grow high enough to reach the filter container.Fig.3depicts the progressin the adapted parameter throughout time. As seen in the Fig., effective filtration area remains $100 \%$ during the initial part. However it drops dramatically after a certain point, where the cake height is restricted to grow by the filter container hard wall. The clogging phase after this point cannot be modelled by the methods exists in the literature.

The formula given in (6) cannot be used for prognostics purposes directly. The dynamic rate of change in the pressure drop will be more useful for prognostics purposes. In other words, a dynamic state transition is required for modelling the 
degradation behaviour of the system. If the severity of the filter clogging increases, then the pressure drop changes. Thus, the presented equation is transformed into a dynamic state transition equation to be able to serve for prognostics purposes. The rate of change in pressure drop in sufficiently small ' $d t$ 'time can be formulated to give:

$$
\Delta P_{t+d t} \cong \Delta P_{t}+\Delta P_{t}^{\prime} d t+w_{t}
$$

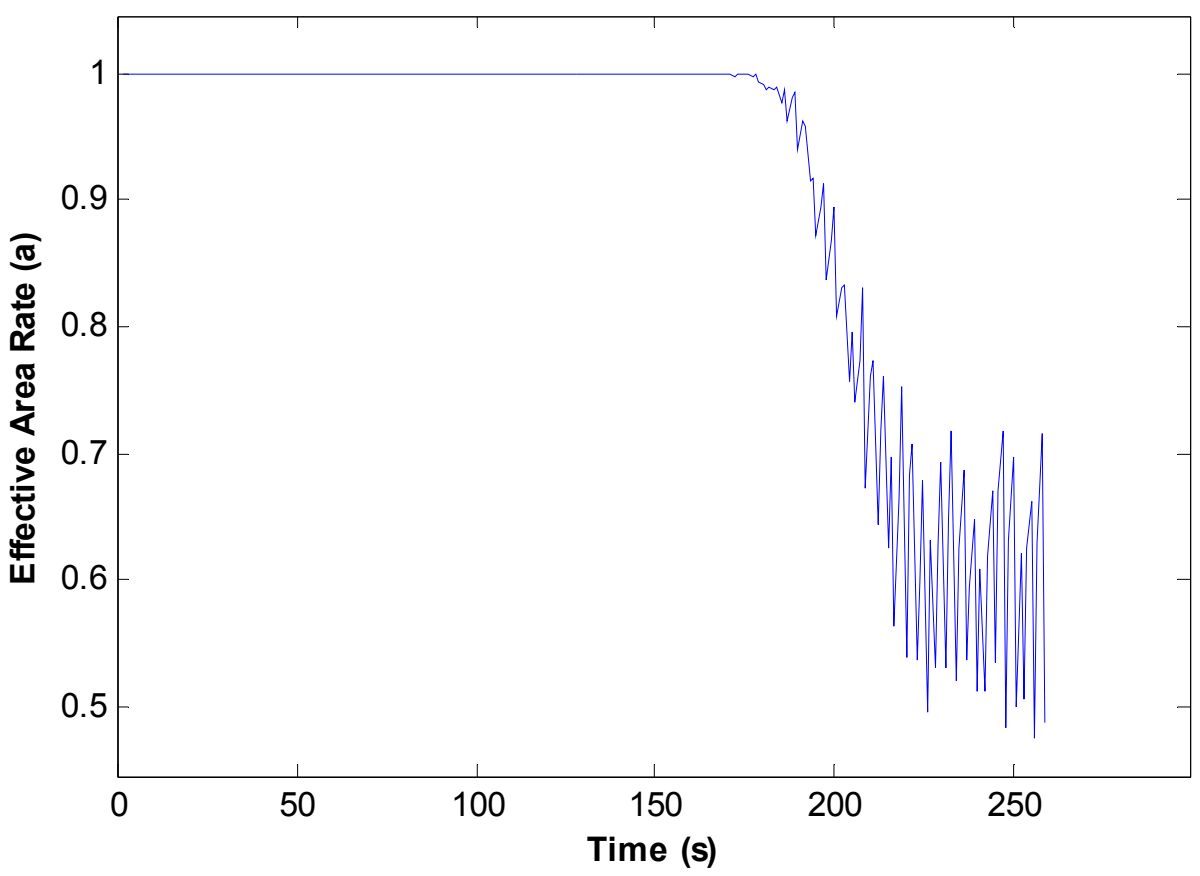

Fig.3: Sphere packing simulation results of the adapted parameter

Eq. (7) represents a nonlinear pressure drop increment steps. $w_{t}$ in the equation represents the process noise whereas ' $\Delta P_{t}{ }^{\prime}$ ' term can be obtained by taking the first derivative of the equation given in Eq. (6):

$$
\Delta P^{\prime}=\frac{10 A V_{s} \mu}{d^{2} a \epsilon^{3}}\left[\frac{\epsilon(1-\epsilon)^{2} L^{\prime}-(1-\epsilon)(3-\epsilon) L \epsilon^{\prime}}{\epsilon}+\frac{(1-\epsilon)^{2} L a^{\prime}}{a}\right]+\frac{B \rho V_{s}^{2}}{d a \epsilon^{3}}\left[\frac{(2 \epsilon-3) L \epsilon^{\prime}}{\epsilon}+\frac{(\epsilon-1) L a^{\prime}}{a}+(1-\epsilon) L^{\prime}\right]
$$

In Eq. (6)-(8) cake thickness ' $L$ ', porosity of the filtration medium ' $\epsilon$ ', effective filtration area ' $a$ ', and the fluid velocity ' $V_{s}$ ' are the dynamic parameters while rest of the parameters remain constant as the filtration process proceeds. In this regard, these dynamic parameters are required to be modelled separately for prognostic goals. It is important to note that, even though the fluid velocity changes over time, we have not modelled the velocity and assumed it to be constant, for simplicity.

Filter clogging severity is identified based on the pressure dropacross the filter. If all the parameters on the right hand side of the Eq. (8) are given, then the pressure drop can be calculated by basically adding the pressure drop increase rate to the previous pressure drop value. The pressure drop is also measured using the sensors installed in the system. The forecasting of the pressure drop is the fundamental issue and the answer to the question of "how the pressure drop (i.e., clogging severity) will progress?" is sought here. The forecasting of the pressure drop will be based on the formula given in 8 that uses measured parameters in the current time.

Cake thickness and porosity are the main dynamic cake structure parameters which are required to be obtained in Eq.6. We have used high quality, continuously captured filter mesh pictures which are taken to measure the cake thickness. Image processing techniques have been used based on the obtained images to correlate particle deposition with cake thickness phenomena.

The other parameter to be measured is the porosity; however in this study no porosity measurements have been collected therefore porosity values obtained during the simulation are not validated. Porosity ' $\epsilon$ ' is defined as the void fraction of a filtration cake. The porosity calculation model is provided in Eq. (9) where ' $M_{c}$ ' is the loaded mass of particles, ' $\rho$ ' is the particle density, and ' $A_{f}$ ' is the cake area. The term ' $M_{c} / \rho$ ' gives the loaded cumulative particle volume for each time 
instance whereas ' $L A_{f}$ ' stands for the cake volume. Loaded particle volume is calculated by multiplying the flow rate (i.e. ' $Q$ ') of the system by the solid fraction (i.e. ' $x$ ') of the suspension.

$$
\epsilon=\frac{\text { void volume }}{\text { total cake volume }}=1-\frac{M_{c} / \rho}{L A_{f}}
$$

\subsection{Particle filters and physics-based modelling}

Kalman and particle filters are two of the most known Bayesian stochastic filtering techniques, which have been widely used in prognostics, object tracking, computer vision and robotics, speech recognition; and in general, machine learning. Kalman filters (KF) are limited to the occasions where the degradation of an asset exhibit linear characteristics. KF estimators approximate the parameter distributions of the model, deterministically. On the other hand, in particle filters (PF), model parameter distributions are represented by means of significant amount of weighted particles rather than an analytic probability distribution function (PDF) [27]. This means that each particle contributes to the parameter probability distribution and evolves through time. In addition, PFs are more generic compared to KFs, hence they are applicable to nonlinear degradation profiles and also are not limited to the Gaussian noise. Therefore, in this study, we have selected PFs over KFs as they provide wider application space for filter clogging modelling. A more detailed discussion on PF's mathematical background can be found in the literature [29], therefore a brief discussion of PF applications in prognostics are provided as follows.

Particle filters, also called as 'Sequential Monte Carlo Estimators', have been used widely in prognostics, peculiarly integrated in physics-based models. Some of the examples found in the literature are; fatigue crack propagation modelling for various engineering structures [28], [29], [30], [31], [32] and [33], battery capacity modelling [29],[34] and[35], centrifugal pump degradation modelling [36], thermal processing unit degradation [37], pneumatic valve modelling [38], DC-DC converter system level degradation modelling [39], Isolated Gate Bipolar Transistor (IGBT) degradation modelling [40], Proton Exchange Membrane Fuel Cells (PEMFC) life modelling [41], Lumen degradation modelling for LED light sources [42]. The list can be expanded to various engineering prognostic applications.

In general, dynamic systems can be modelled in the form of state transition equation, which describes the evolution of its state through time [31]. The system state and measurement models underpinning Particle Filter process are given in Eq. (10 and11). System state model represented in (10) formulates the state of the system at time $k$ based on the system state at time $k-1$. In other words, the future progression of the states is estimated based on the current state. The filter clogging formula given in Eq. (8) will be used as the state transition equation.

$$
\begin{aligned}
& x_{k}=g_{k}\left(x_{k-1}, \theta_{k-1}, w_{k-1}\right) \\
& z_{k}=h_{k}\left(x_{k}, v_{k}\right)
\end{aligned}
$$

where $g_{k}: R^{n_{x}} \times R^{n_{\theta}} \times R^{n_{w}} \rightarrow R^{n_{x}}$ is the dynamic state transition equation, $x_{k}-x_{k-1}$ is the state vector at discrete time points $\mathrm{k}$ and $\mathrm{k}-1, \theta_{k}$ is the model parameter vector, $w_{k}$ is the process noise, $h_{k}: R^{n_{x}} \times R^{n_{v}} \rightarrow R^{n_{z}}$ is the measurement equation, $z_{k}$ is the measurement at time point $\mathrm{k}$, and $v_{k}$ is the measurement noise.

Particles, evolving in the system, can be represented as: ' $\left\{x_{k}^{i}, \theta_{k}^{i}, w_{k}^{i}\right\}_{i=1}^{N}$ ', where ' $N$ ' symbolises the total number of particles and ' $i$ ' is the particle index. Each particle accommodates a clogging state variable ' $x$ ', model parameters ' $\theta$ ', and a process noise value ' $w$ ', which evolve through time. This means that the filter clogging severity degradation distribution will be constructed with $\mathrm{N}$ number of different particles. Generally, the higher number of particles used in the construction of parameter distribution, the better representativeness of the system. Therefore, we selected a reasonably high number for ' $N$ ' in the modelling of filter clogging. However, excessively higher numbers for ' $N$ ' will increase the computational complexity, which may be burdensome when dealing with higher numbers of system parameters. The model parameters are symbolised in ' $\theta$ ' which encapsulates the Ergun Equation parameters ' $A$ ' and ' $B$ '. ' $A$ ' and' $B$ ' are system specific constants which are required to be learned via a system measurement feed. ' $x$ ' and ' $z$ ' are the state variable and measurement values, respectively. ' $x$ ' values represent the pressure drop state predictions obtained from the state transition equation. On the other hand, ' $z$ ' values stands for the pressure drop noisy sensor measurements. Note that, a separate particle filter is employed to track the cake thickness trend against time which has its own state and measurement variables. Since the mechanism is the same, authors avoided repetition of explaining the same process.

In particle filters, the posterior distribution filtering process usually comprises three recursive steps: (1) Prediction, (2) Update, (3) Resampling. The steps of the simulation algorithm are illustrated in Fig.4. In the prediction step, system state is 
predicted using previous step's the updated parameters via state transition equation. Then the predictions are updated for the current time step by using a likelihood function shown in Eq. (12). Likelihood function assigns weights to particles according to the closeness to the measurement at each time point. In the resampling step, the particles with lower and higher weights are eliminated andduplicated, respectively, which is called inverse CDF (cumulative density function) method [29]. This filtering process is entitled as Sequential Importance Resampling (SIR) particle filters.

$$
L(z \mid x, \theta, \sigma)=\frac{1}{\sqrt{2 \pi} \sigma} \exp \left[\frac{1}{2}\left(\frac{z-x(\theta)}{\sigma}\right)^{2}\right]
$$

This parameter learning process is continued until no measurements have left where the extrapolation step commences (i.e. actual RUL calculation step). In the extrapolation phase where the parameter learning has stopped, the state parameter vector (i.e. ' $x$ ') is projected continuously by using the state transition equation (with the fixed parameter distributions) until

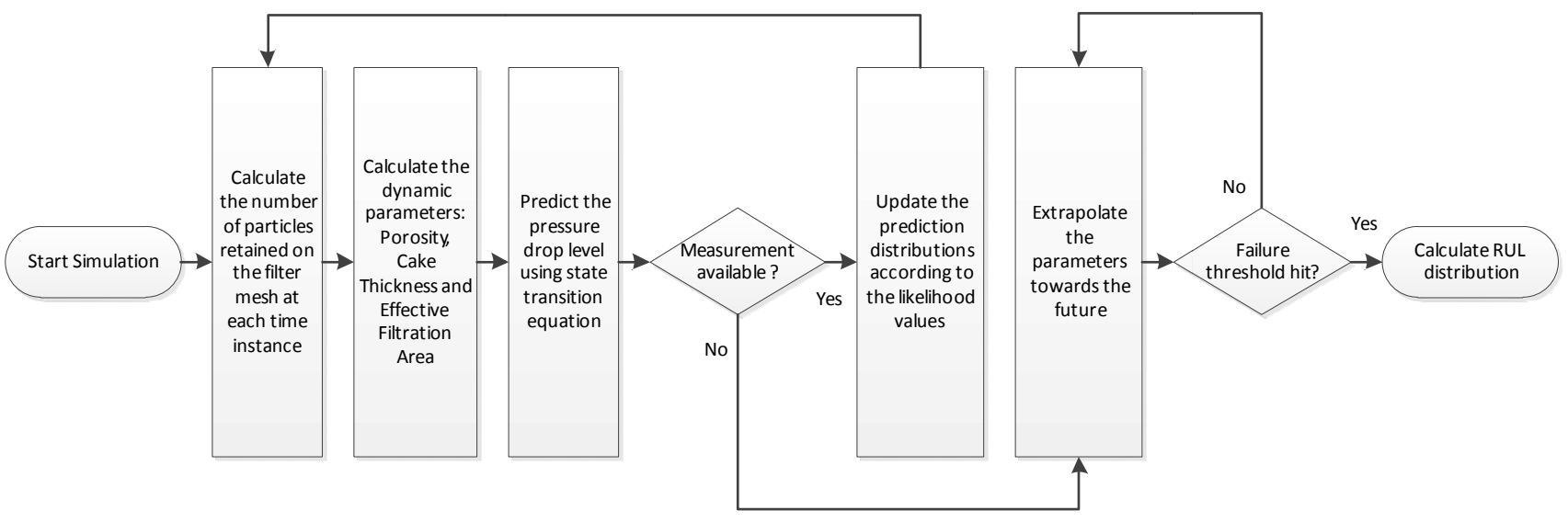

Fig.4. Flowchart of the RUL calculation

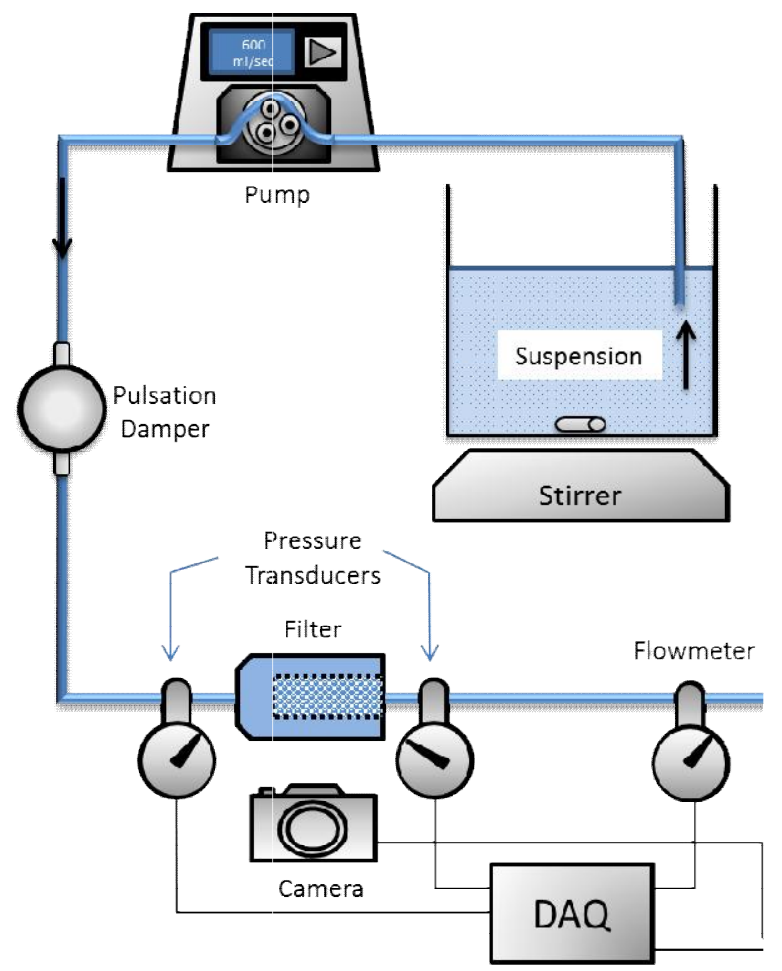

Fig.5: Filter clogging prognostic rig system design

itreaches the failure threshold. In this way, ' $N$ ' number of trajectories also entails the distribution of RUL estimations. Mean or median of the RUL distribution is generally used for visualisation of the estimated RULs. 


\section{Results}

This chapter includes four subsections. The first one presents the design of the experimental rig to be used for data collection for filter clogging phenomena. The second subsection discusses measurement of parameters from the experimental rig. The third subsection presents the data collection procedures. The last subsection presents the prognostics results obtained from the methodology presented in the previous chapter using the collected data.

\subsection{Experimental Rig Design}

An experimental rig to demonstrate filter clogging failure should consist of the following major components: Pump, liquid tanks, tank stirrer, pulsation dampener, filter, pressure and flow rate sensors, data acquisition system connected to a computer. Error! Reference source not found.illustrates the design of such experimental rig. The prognostic rig is designed so that no other component will deteriorate other than the filter during the data collection process. This means that, filter clogging is the only failure type to be targeted in the degradation modelling. Each component is discussed below.

4.1.1 Pump: There are different types of pumps enabling a liquid to flow through a complex system. Since the system will involve contaminants in the fluid, a peristaltic pump has been used as its mechanism is more tolerant to particles in the liquid.

A Masterflex® SN-77921-70 (Drive: 07523-80, Two Heads: 77200-62, Tubing: L/SC 24) model peristaltic pump was

installed in the system to maintain the flow of the prepared suspension. The pump is a positive displacement source, providing a flow rate ranging from 0.28 to $1700 \mathrm{ml} / \mathrm{min}$ (i.e. from 0.1 to $600 \mathrm{RPM}$ ). The practical part of peristaltic pumps is that they confine the fluid to the tubing. In this way, the pump cannot contaminate the fluid and vice versa. Detailed design of the prognostic rig is illustrated inError! Reference source not found.. A photograph of the test system capturingall components is displayed in

Fig.7.

4.1.2 Dampener: The aim of using rigid tubing is to prevent the system from the unwanted tubing expansion due to pressure build up which interrupts the actual pressure build up generated from filter clogging. A Masterflex ${ }^{\circledR}$ pulse dampener is installed on

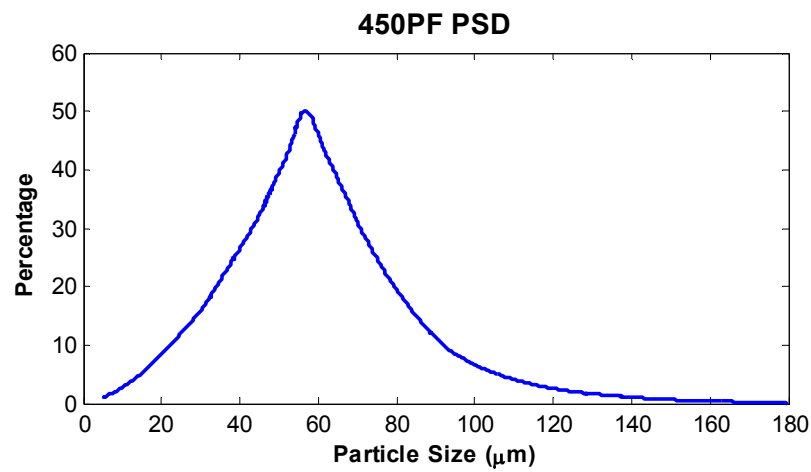

Fig.6: PEEK particle size distribution

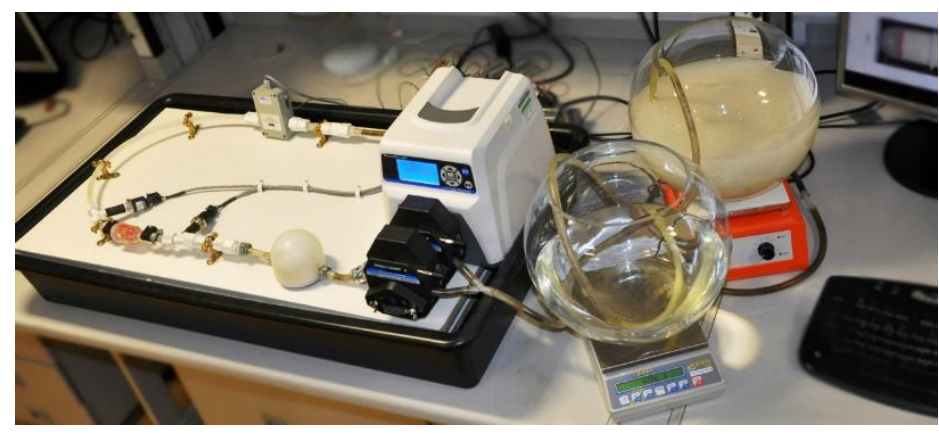

Fig.7: Filter clogging prognostic rig 
the downstream side of pump to eliminate the pulsation in flow, hence pressure drop across the filter. Majority of the system is furnished with a rigid polypropylene tubing whereas the pump side is covered with a flexible Tygon ${ }^{\circledR}$ LFL pump tubing.

4.1.3 Tank: One half-sphere-shaped main tank and two subsidiary tanks (i.e. reservoir tank and clean water tank) are installed in the system. The sphere shape tank bowl enables the stirrer work efficiently leading to homogeneously distributed slurry in the tank. The prepared suspension is kept in the main tank and pumped through the filter and poured into the reservoir tank. The clean water tank is used to fill-up the system components (e.g. tubing and the filter chamber) with clean water prior to each test. A Kern ${ }^{\circledR} 10000-1 \mathrm{~N}$ type high precision weighing scale (weighing range: $0.1-10,000$ g.) is placed under the reservoir tank and connected to the PC with a serial cable to keep track of the amount of filtrated liquid continuously.

4.1.4 Particles: The suspension is composed of Polyetheretherketone (PEEK) particles and water. PEEK particles have a density $\left(1.3 \mathrm{~g} / \mathrm{cm}^{3}\right)$ close to that of room temperature water and have significantly low water absorption level $(0.1 \% / 24 \mathrm{~h}$, ASTM D570). Having a low water absorption level will prevent particles to expand their volume when they mix with water. Subsequently, closer density with water allows particles to suspend longer in water. Therefore, PEEK particles are selected to be used in the accelerated clogging of filter experiments. The particles have a large size distribution as seen inFig.6. For this reason, narrowing the distribution by sieving is found to be necessary before conducting experiments.

4.1.5 Stirrer: An adjustable speed ceramic SC-1 type magnetic stirrer was installed in the system to ensure that the particles are distributed uniformly in the tank during the experiments. This is necessary as the particles, even though they are meant to be naturally buoyant, sink after a while leaving the water clean.

4.1.6 Pressure Sensors: Upstream and downstream Ashcroft ${ }^{\circledR}$ G2 pressure transducers (measurement range: 0 - 100 PSI) are installed in the system to capture the pressure drop (i.e. ' $\Delta P$ ') across the filter, which is considered as the main indicator of clogging.

4.1.7 Flow Rate Sensor: A GMAG100 series electromagnetic flow metre (measurement range: $3-25,000 \mathrm{ml} / \mathrm{min}$ ) is installed in the system to keep track of the flow rate in the system. The flow metre is also suitable for high pulsation flows. Magnetic flowmeters have no moving parts, which allow measuring the flow rate of slurry by means of the magnetoinductive principle. This type of flow meters has been selected for two reasons: (1) To enable measuring flow rate of water \& PEEK suspension with no accuracy degradation; (2) They are reliable and entitled with low unnecessary pressure loss levels across the flow metre. In addition, a pulse rate to current converter is interfaced with the flow meter for converting frequency to proportional analogue 4-20mA current outputs.

4.1.8 Camera: A high quality macro lens camera is positioned on top the filter chamber, enabling to take macro pictures every two seconds. The mesh inside the filter; hence, the retained particles can clearly be captured and used in an image processing application for determining the ground truth clogging rate or an auxiliary source for modelling of the filter clogging phenomena. To be more precise, pressure and flow rate data can be compared or utilised with the features extracted from the macro picture data.

A box was designed to cover the filtration area. The interior side of the box was masked with a white coloured material where a light source was projected inside the box to provide a constant uniform light so that the filter is isolated from varying environmental light. All components are placed on a grid style dripping tray in order to prevent potential problems due to a potential leakage.

\subsection{Obtaining parameters}

As discussed in the previous sections, cake thickness and porosity are the two main parameters to be measured or modelled during the continuous monitoring. Porosity calculation is discussed in the methodology section. This section discusses the details of obtaining the cake thickness.

As mentioned in the methodology section, camera and image processing techniques are used to measure the cake thickness. Fig. 8 demonstrates the measurement of cake thickness information. Original and the black and white transformation of the filter picture are depicted. Image processing was performed on the orange rectangular area covering one of the filter meshes. An image processing programme is developed to capture the biggest white area (i.e. highlighted in green lines) within the orange zone. The reference line is located in the far left of the mesh area. It is assumed that the cake thickness is directly proportional to the expansion of particles to the left, starting from the reference point. Therefore, the average expansion rate is calculated each second during the experiments, as illustrated in Fig.9. 
In Fig.9, the blue dots represent the average cake thickness values obtained from the picture data via the image processing programme. Black solid line stands for the maximum cake thickness level restricted by the filter container.

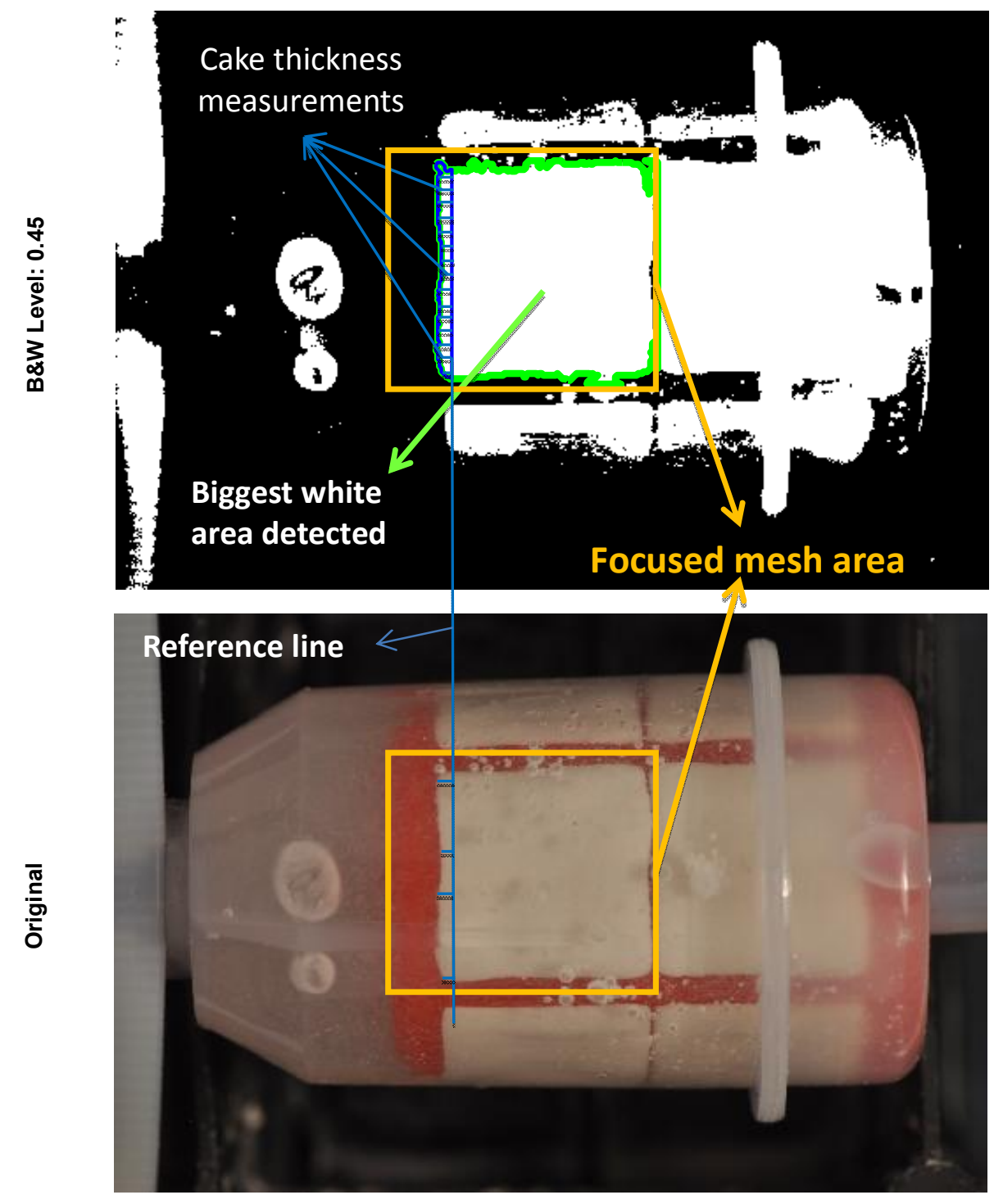

Fig.8: Cake thickness calculation using filter images 


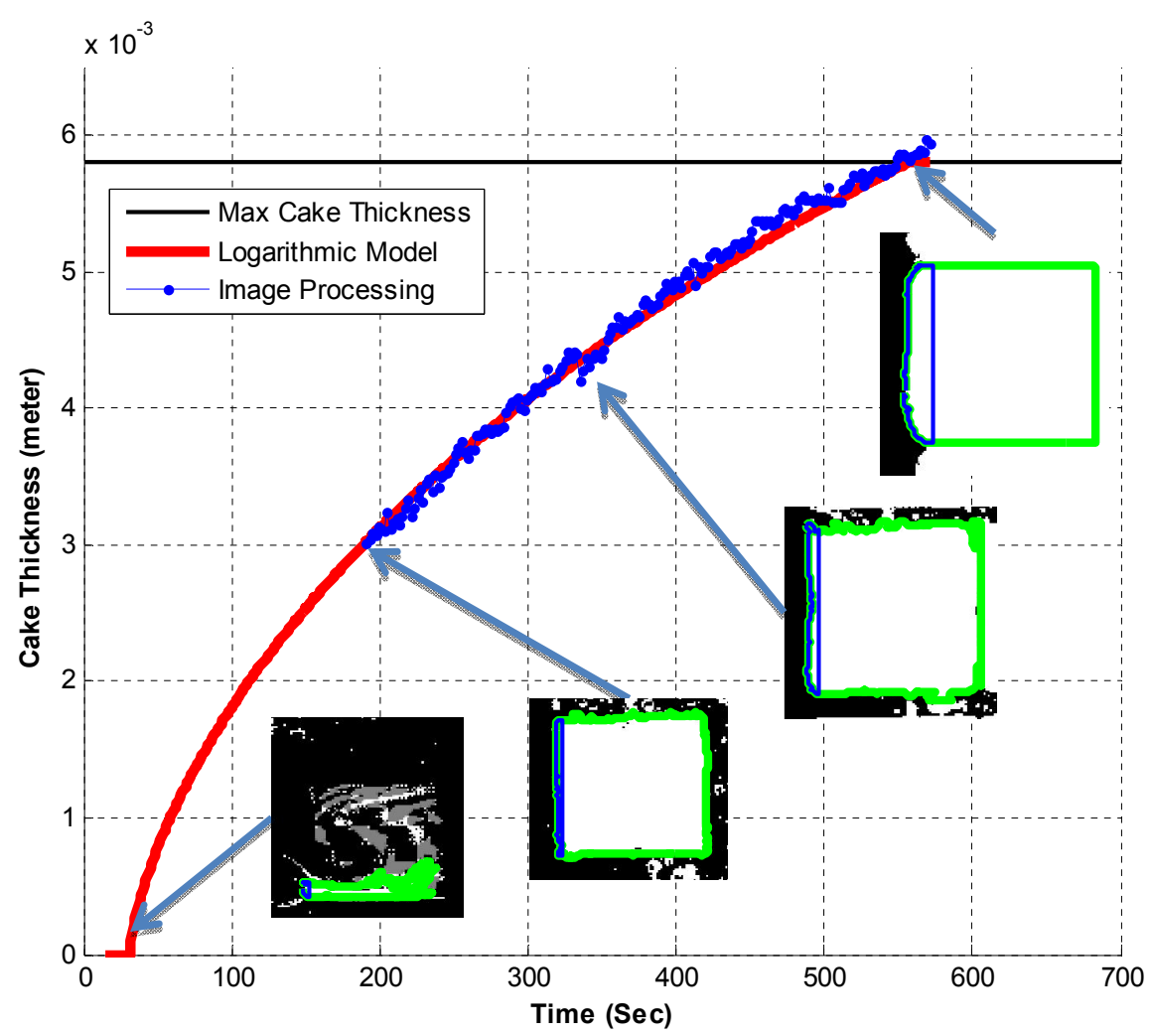

Fig.9: Cake thickness modelling demonstration

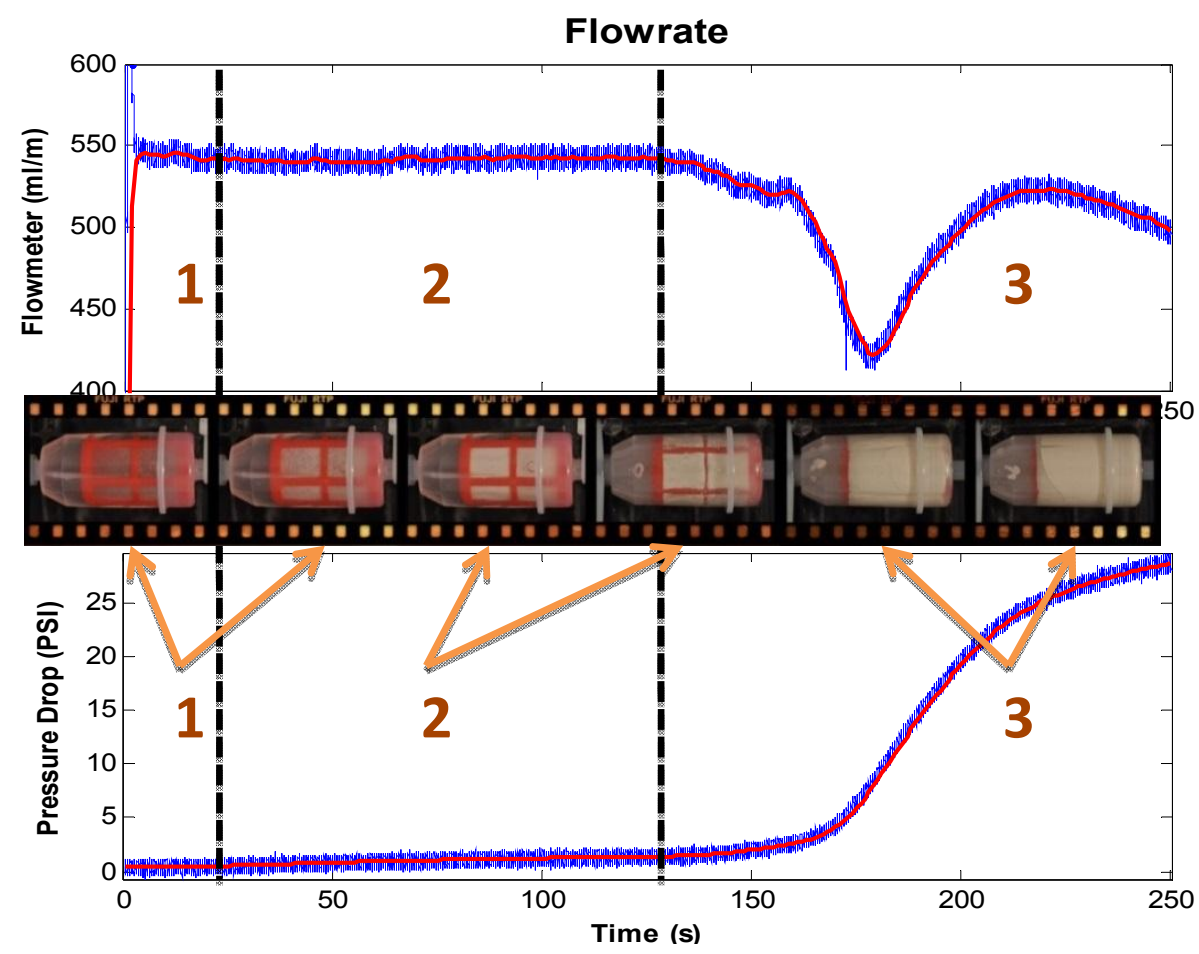

Fig.10: Filtration phases

In addition, the empirical logarithmic cake thickness measurement model is shown in solid red line. The measurement model is obtained by fitting a logarithmic growth trajectory to the indirect cake thickness measurement points obtained from the image processing technique. The pressure drop data is also utilised to define the minimum and maximum cake thickness time point detection. The empirical cake thickness model is taken as the final cake thickness measurement and used as auxiliary information in pressure drop modelling. 
The three phases of the filter clogging is displayed in Fig.10. Noting that all these phases assumed to fall under the constant rate filtration phenomena discussed in the literature review section. The first phase represents so called 'clean filter filtration stage' which is the predecessor stage of the actual cake filtration [9]. In this phase, majority of the particles passes through the filter mesh without being retained, however bridges may appear to form by jamming of the particles gradually. During this phase, pressure and flow rate values remain relatively constant. At the end of this phase, filter medium pores are blocked which led to dramatic increase in the retention rate of particles. Second phasecan be called 'actual cake filtration' as the captured particles form and build up the layers of cake which is significantly prolonged step than the initial one. The pressure drop increases steadily while flow rate remains constant. As soon as the cake thickness reaches the filter container interior level height, a sudden drop occurs in flow rate measurement whereas the pressure drop values enter to an exponentially growing region. This dramatic increase in pressure drop is thought to be by virtue of the restriction of cake thickness by the filter chamber which led to raise different type of forces (e.g. reduction in effective filtration area). However, the growth in pressure drop turns into logarithmic characteristics as the pump approaches its maximum pressure levels.Note that the third phase presented in this paper has not been modelled in the literature before.

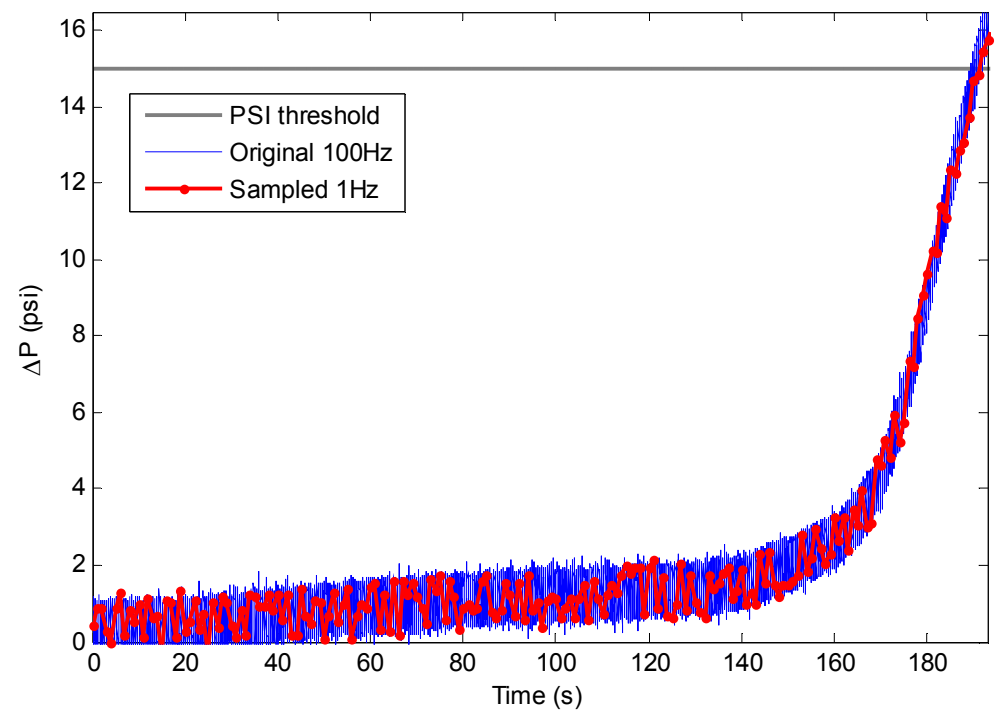

Fig.11: Original $100 \mathrm{~Hz}$ vs $1 \mathrm{~Hz}$ down-sampled data for filter clogging dataset

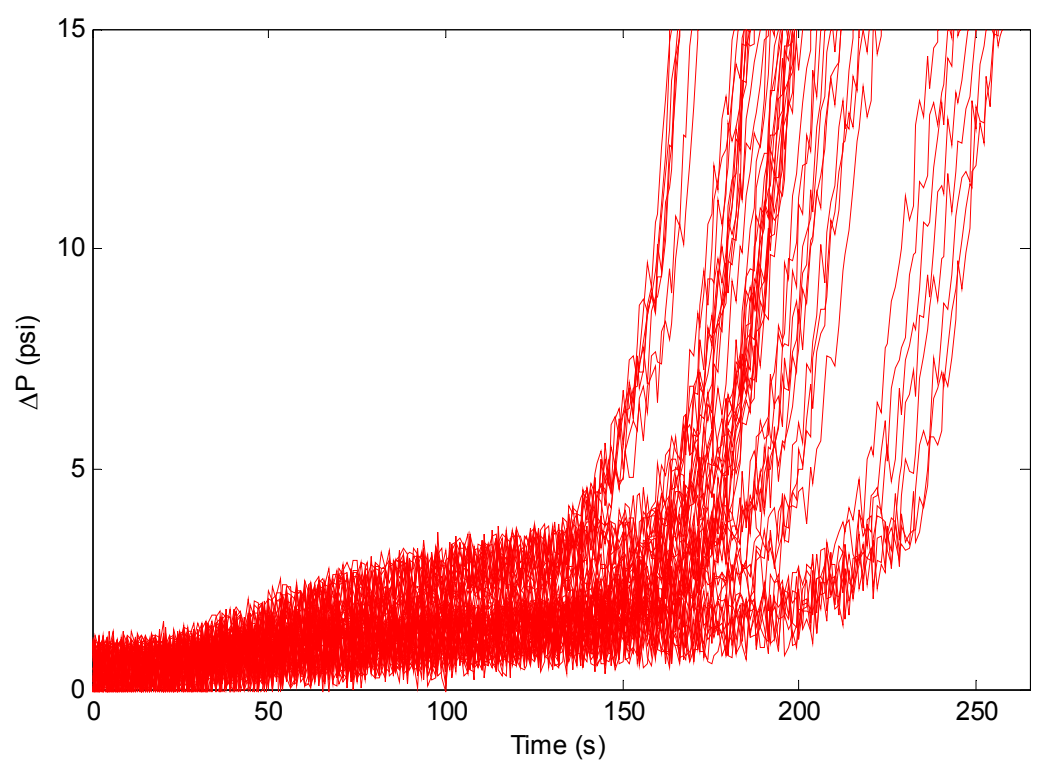

Fig.12: The final pressure drop trajectories for filter clogging dataset 


\subsection{Data collection}

This study involves an experimental test rig setup to produce a prognostic benchmark dataset. The dataset consist of 56 run-to-failure samples obtained from well-controlled accelerated filter clogging experiments. The previous attempts of data collection for filter clogging failure scenario can be found in [43] and[44]. The improvements in the system design and data collection mechanism are resulted in the collection of reproducible and well-organised dataset. A brief summary of the data collection mechanism is provided as follows.

Before the actual data collection, several errands are required to be conducted. The PEEK polymer particles, representing the contamination in liquid to be purified, are sieved to narrow the particle size distribution. Therefore, after the sieving, four different groups of particles with different size distributions are obtained. Particles are sieved into 45-53, 53-63, and 63-75 micron range groups. In addition, auxiliary tests with clean water are conducted prior to each run-to-failure experiment. The necessity for these preliminary tests is to dispose air bubbles within the system which will ease the modelling of clogging process. Furthermore, these preliminary runs are also useful for calibrating the system parameters before the actual tests.

In addition to different particle size distributions, we have tested different rates of solid fractions in the suspension. Four different solid ratios are determined, ranging from $0.400 \%$ to $0.475 \%$ levels. As a result, data collection has been conducted for sixteen different operational profiles each of which have four samples. Exceptionally, the last four profiles have fewer samples compared to rest of the profiles. Therefore, the operational profiles created are the outcomes of predefined combinations of particle size distribution and solid ratio levels of the suspension.

The tests have been conducted by setting the pump with 211 RPM to produce $600 \mathrm{ml} / \mathrm{min}$ flow rate initially. The pressure and flow rate readings have been collected continuously as they are the main indicators of clogging. Each clogging experiment has been conducted and monitored until the filter has clogged up where the pressure drop value has reached its peak and entered into a stable pressure region. The sample rate for the data collection is kept $100 \mathrm{~Hz}$. However, for the modelling studies, the signals are down-sampled to $1 \mathrm{~Hz}$ as shown in Fig.11. In the Fig., the original signals are represented in blue whereas the sampled signal plotted in dotted red curve.
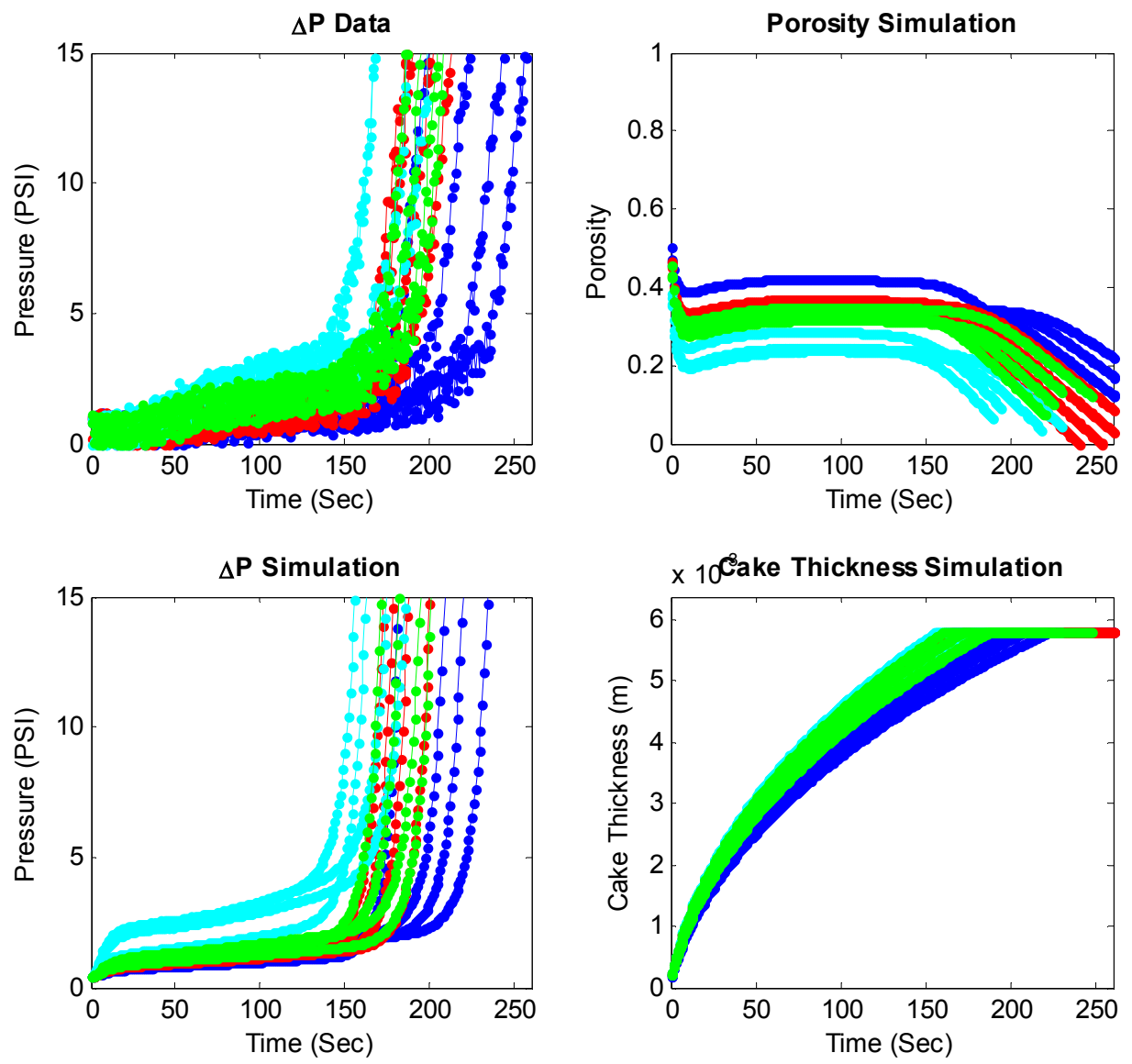

Fig.13: Simulation results prior to particle filter integration 


\subsection{Prognostics results}

A detailed discussion on the filter clogging modelling is provided in the previous sections. In this section, we present the prognostic modelling results of the filter clogging experimental study. This section also entails the analysis of model performance evaluation.

The pressure drop model, discussed in the Methodology, employs the 1 Hz sampled data in order to ease the computational time concerns. The final look of the entire sampled dataset signals are visualised in Fig.12. It is evident in the Fig. that the signals have an intrinsic noise. If we are to explain the variation within the dataset from top-down level, the threshold hitting spread (i.e. 36\% variation among the samples) is due to the operational profile input variation (i.e. 16 different operational profiles). For each set in the same operational profile, the variation is measured approximately $3 \%$. The final uncertainty to be considered in the particular signal level is the noise due to the pump pulsations. Even though the system design and wellcontrolled operational procedures have minimised this effect, pressure drop signals still have the pulsation, hence the noise in the system.

However, true pressure drop levels can be estimated by using particle filters. The filter clogging physics-based model comprising of an analytic pressure drop equation and a particle filter mechanism is discussed in the previous section. Briefly, the well-known Ergun equation is discretised and integrated with particle filters to give a dynamic degradation modelling solution for filter clogging (i.e. Eqs $(7,8)$ ).

Sphere packing theory discusses how non-overlapping spheres are arranged to fill a space that may model the physics of cake thickness and porosity in clogging. The dynamic parameters in the equations such as cake thickness and porosity are simulated via utilising the sphere packing theory. Then, the parameters obtained are embedded into particle filtering.
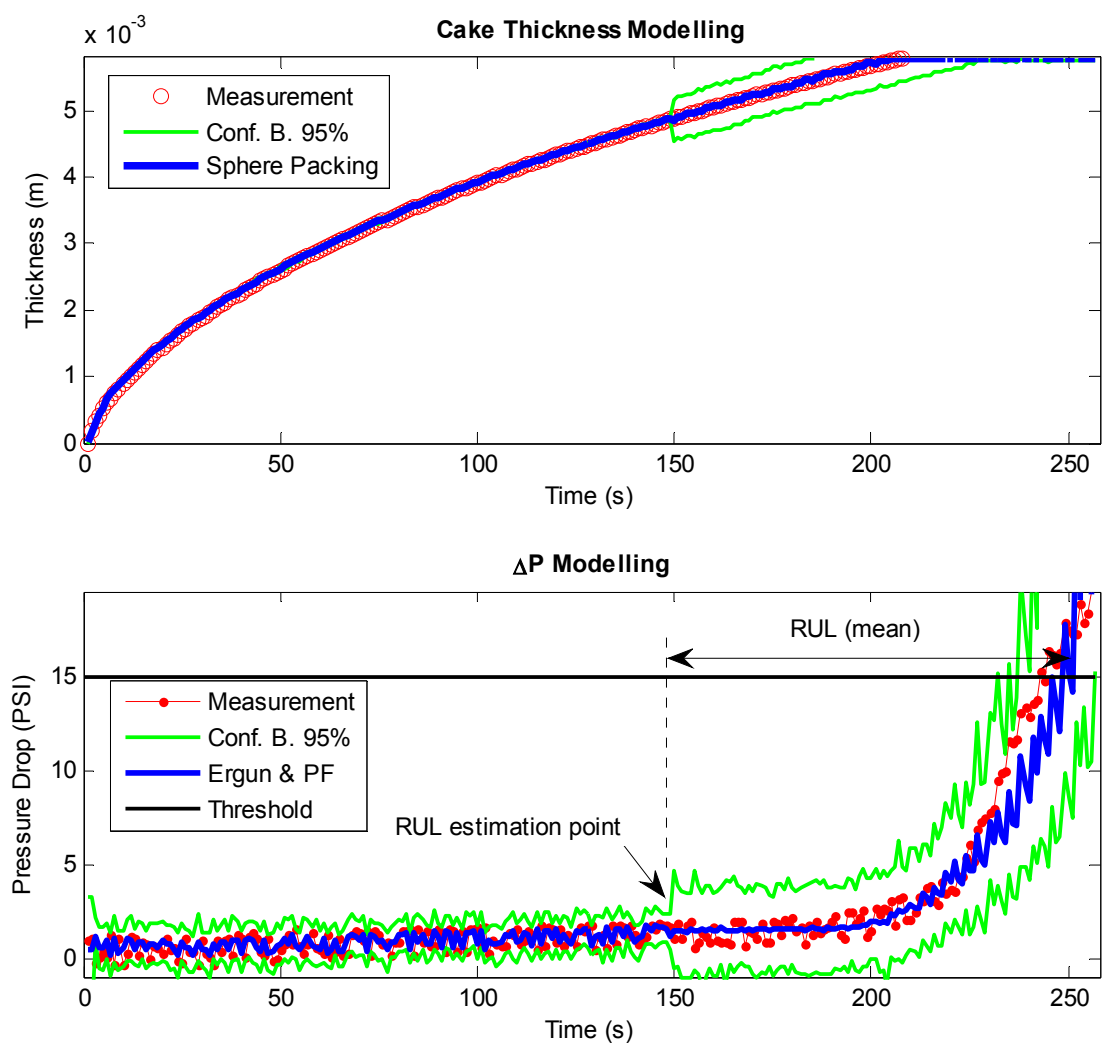

Fig.14: Cake thickness and pressure drop modelling

Simulation results prior to particle filter integration are displayed in Fig.13. In the Figure, top left plot exemplify the actual pressure drop data for different particle size and solid ratio combinations whereas the rest visualises the dynamic parameter simulation outputs. Each colour represents a different particle size distribution category. To be more precise, the blue, red, cyan and green colours represent the 45-53, 53-63, 63-75micron range and the original particle size distributions respectively.

Two separate particle filter mechanisms are integrated into the simulations. The first one tracks the sphere packing cake thickness model whereas the latter tracks the Ergun pressure drop model and its parameters. The standard deviation values 
selected for the measurement ' $\sigma_{v}$ ' and process noise ' $\sigma_{w}$ ' are 0.01 and 0.001 respectively. Five hundred numbers of particles are employed for particle filters. Fig.14 illustrates the demonstration of particle filter mechanisms integrated in the cake thickness and pressure drop modelling. For this demonstration, the parameters are learned and updated until 150th second throughout the sample lifetime. Starting from the RUL estimation point, where the measurement input feed is terminated, the model parameters are extrapolated towards the future up to the maximum pressure drop threshold level using the discretised Ergun equation with Monte Carlo simulation. Thus, the RUL distribution at this specific point is obtained by calculating the differences between the RUL estimation starting point time and the times where trajectories (i.e. the number of trajectories is equal to the number of particles) hit the threshold for the first time. In the Fig., blue lines represent the median values of the distribution whereas the green curves encapsulate the $95 \%$ of the spread within the distribution (i.e. confidence bounds).

For each test specimen, RUL estimations are set to perform at every five seconds. The RUL prediction results are visualised in Fig. 15 where the results obtained from 16 test samples are shown. Each test sample is a representative of its operational profile. For theseFig.s, the $4 \times 4$ matrix plotting mechanism is organised so that the rows represent the particle size distributions while the columns indicate the solid ratio levels. For instance, Sample 42 belongs to the eleventh operational profile where the particle size distribution is in the 63-75 micron range and the solid ratio for the suspension is $0.45 \%$.

In Fig.15, x-axes scales the life duration of a specific sample whereas y-axes stands for the corresponding RUL values. In this Fig., the dashed linear black lines represent the actual RUL values. Actual RUL values for a specimen are calculated by subtracting the current cycle from the end-of-life $(\mathrm{EoL})$ value specific to the specimen. The dotted blue lines represent the physics-based prognostic model (i.e. Ergun equation and Particle Filters). In addition, the green dashed curves stands for the confidence bounds for the predictions where they encapsulate the $95 \%$ of the RUL distribution. If we are to analyse the Fig. visually, one can say that the predictions remain significantly close to the actual RUL values for majority of the samples. However, inconsistent RUL estimations are obtained for Sample 34. This case is assumed to be an outlier. The closer to actual (real) RUL values the better prognostic results. The distance in between the mean RUL prediction and the actual RUL value considered as the error. The ultimate aim is to produce prognostic result with minimized error levels. However, the significance of an error may vary during a degradation process. Therefore, it is necessary to briefly discuss the prognostic performance metrics before investigating the prognostic performance for this study.

Saxena et al. [45] claims that traditional forecasting performance metrics such as 'root mean squared error' (RMSE) and 'mean absolute deviation' (MAD) do not perfectly accommodate prognostic model performance requirements. For instance 

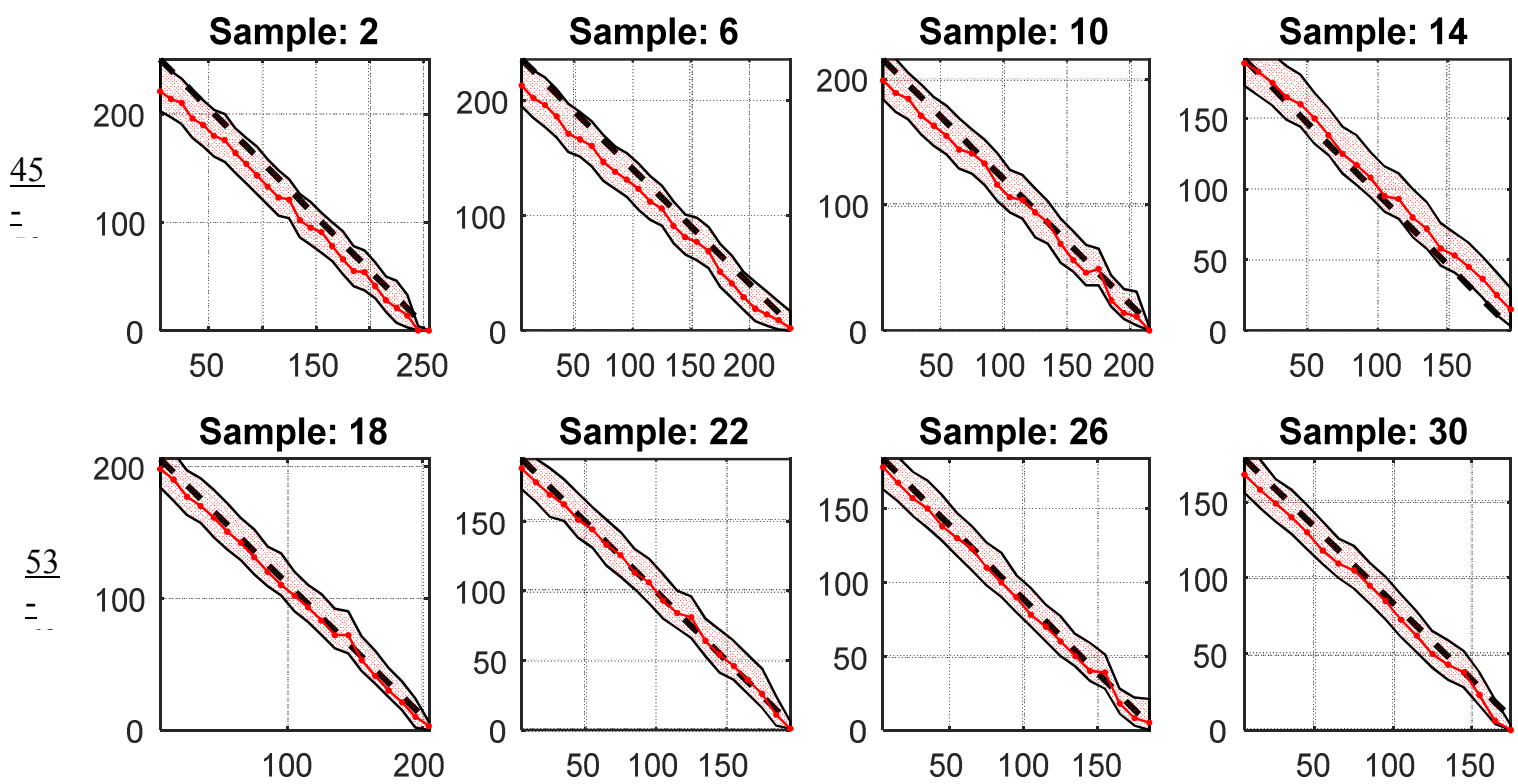

Sample: 22
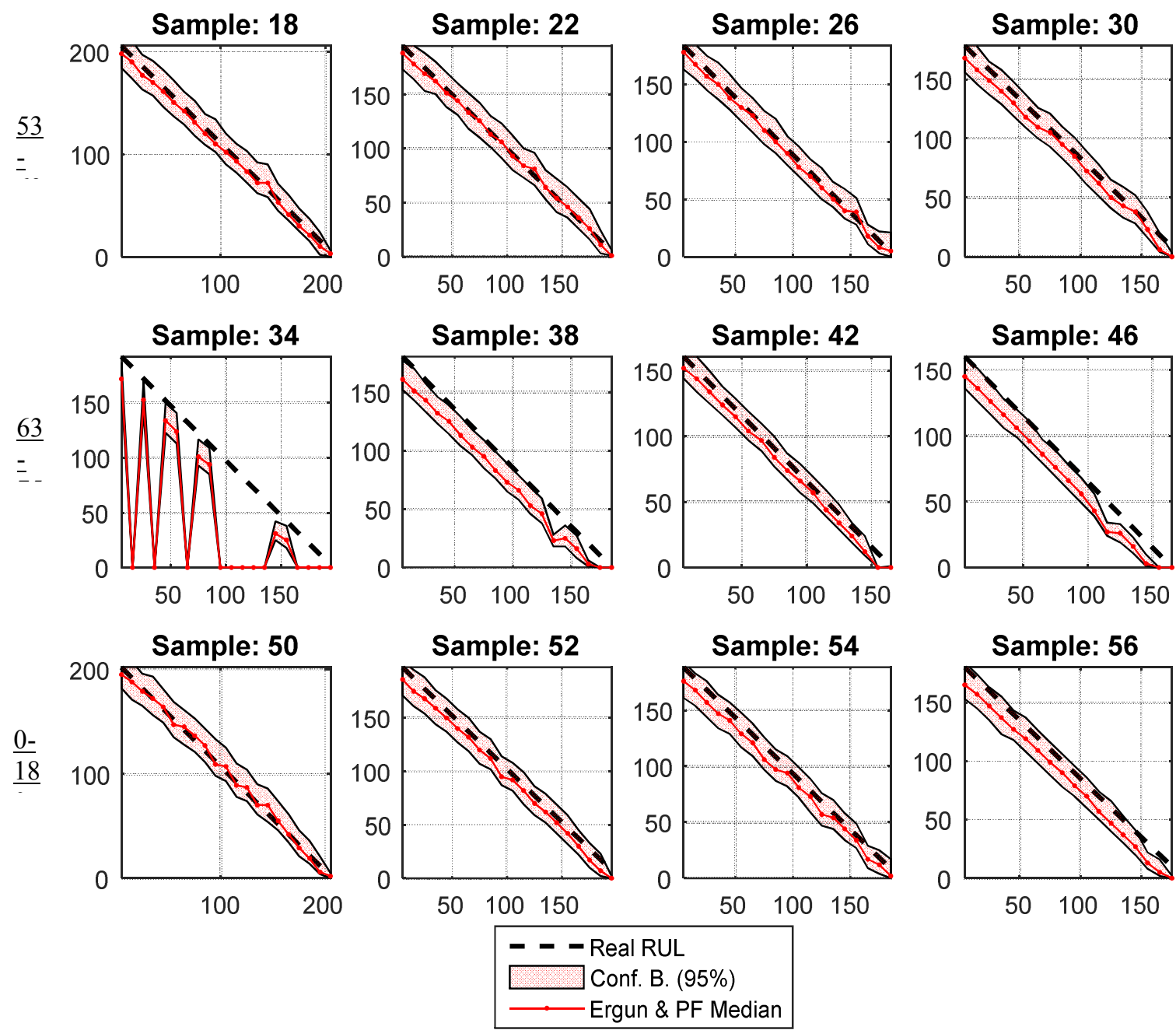

Fig.15: Physics-based modelling RUL results

these metrics are not designed for applications where the predictions are updated continuously as more data become available. Typically, prognostic prediction performance tends to improve as time progresses where the asset nears its end-oflife. In the early stages of an equipment degradation process, predictions are anticipated to be less accurate since there are not enough measurements fed to update the model parameters. Therefore, penalty rates of the crucial time points for errors should be higher than the earlier stages. Certainly, it is found to be necessary to tailor these traditional prediction performance metrics for prognostic algorithm performance evaluation. A research group from NASA have been conducting a comprehensive research on the standardisation of prognostic evaluation metrics [45], [46]. [47], [48] and [49]. They have introduced a hierarchical group of prognostic evaluation metrics. In this hierarchical design, a prognostic algorithm resultsare tested and passed to the next metric if the metric condition is satisfied. The new metrics designed for prognostic is listed as follows: 
Table 1:

Prognostic performance results

\begin{tabular}{|c|c|c|c|c|c|}
\hline & \multicolumn{5}{|c|}{ Evaluation Method } \\
\hline & $\mathrm{PH}(\%)$ & $\alpha-\lambda(\%)$ & CRA (\%) & Convergence & nRMSE (\%) \\
\hline Metric Value & 94.91 & 53.43 & 79.03 & 0.51 & 7.10 \\
\hline
\end{tabular}

1. Prognostic Horizon (PH)

2. $\alpha-\lambda$ performance

3. Relative Accuracy (RA)

4. Convergence

$\mathrm{PH}$ is defined as the range in between the point where the predictions fall under the allowable error bound (defined by ' $\alpha$ ') for the first time and the end-of-life time point. In other words, PH determines how far in advance an algorithm can provide estimations within the predefined accuracy bounds. Higher $\mathrm{PH}$ values imply longer prognostic horizon, hence better prognostic results. Best possible score for the $\mathrm{PH}$ is that the predictions always stay within the error bound whereas the worst score indicates it never enters the accuracy zone. PH ranges can be described in percentage levels too. We prefer to present $\mathrm{PH}$ results as the percentage of actual life of test specimens.

The $\alpha-\lambda$ performance metric determines whether the predictions fall within the shrinking accuracy cone (defined by ' $\alpha$ ') around the actual RUL values. The output of the metric is binary; however, it can be converted to percentage values if the metric is implemented at multiple time instances. Shrinking cone boundaries are determined by the accuracy modifier ' $\alpha$ '. On the other hand, the parameter ' $\lambda$ ' specifies the rate of actual RUL over full life at time of the first predictions made within the allowable range.

RA is similar to the alpha-lambda accuracy measure. Instead of inspecting whether the predictions fall within the boundaries, RA measures the accuracy level utilising absolute percentage error. Cumulative relative accuracy (CRA) is the weighted average of the RA values for the time instances of prediction points. It is desirable obtaining higher RA and CRA scores for improved prognostics.

Finally, the convergence is the final metric to be verified in the hierarchical design. Firstly, an accuracy or a precision metric such as RA or RMSE is selected. Formerly, the algorithm quantifies whether the accuracy or the precision metric improves over time to converge the true RUL path.

Table 1:

Prognostic performance results provides average values of the performance evaluation results obtained from the sixteen filter clogging experiments tested. In addition to the new prognostic evaluation metrics (i.e. PH, $\alpha-\lambda$ performance, CRA, and convergence), normalised root mean squared error (nRMSE) results are also included in the comparison table. nRMSE metric results are obtained by normalising the RMSE results with mean EoL in the relevant conditions. Thus and so, the nRMSE results can be read as percentage level errors. Higher percentages indicate better prognostic accuracy in prognostic horizon (PH), ' $\alpha-\lambda$ ' performance, and cumulative relative accuracy (CRA) metrics. On the contrary, lower percent nRMSE values and lower convergence distances signify higher accuracy. For all metrics ' $\alpha$ ' and ' $\lambda$ ' values are selected as $0.1(10 \%)$ and 0 respectively.

For PH metric, 96\% value implies that the model stays within the allowable error bound almost all of its life duration. This means that the proposed model can provide accurate estimations starting from fifth percent of filter total life in average. Typically, ' $\alpha-\lambda$ ' performance metric provide binary outputs. However we proportion the number of positive outputs to lifetime percentage levels. Therefore, the results show that $60 \%$ of the model predictions fall into the shrinking $10 \%$ (i.e. ' $\alpha=0.1$ ') error bounds at the time predictions made (i.e. ' $\lambda=0$ '). CRA results indicate that the accuracy level is roughly 
$85 \%$. On the other hand, normalised RMSE results indicate that the error level is $7 \%$ in average. Typically, the convergence metric provides a distance value to represent how fast an algorithm converges to the true values. It is shown in the table that the distance value is significantly low which means the model converges to the true values quickly.

To conclude, the performance evaluation results show that the proposed filter clogging model provide robust and significantly accurate prognostic results.

\section{Conclusions and future work}

Separation of solids from fluid is a vital process to achieve the desired level of purification in many industries. The filter clogging phenomena is the primary failure cause which leads to the replacement of filter or unscheduled maintenance activities caused by a clogged filter.

In this article we present a physics-based prognostic model for the filter clogging phenomena. Differential pressure and high quality filter mesh picture data obtained from an experimental filter clogging test rig, are utilised in the development of the prognostic model. The prognostic performance results show that the prognostic model predict the system behaviour accurately which enables to successfully predict the RUL distribution.

The key areas for the future work will include the investigation of a hybrid integration scheme where the physics-based model and a data-driven model are integrated together. Furthermore, some future work can be expected to further explore the data collection mechanism, test rig design and publication of the collected clogging dataset for prediction and prognostic competitions. Also the dataset can further serve a purpose as a benchmark dataset for prognostic algorithms to be tested on.

It is important to note that, even though the fluid velocity changes over time, we have not modelled the velocity and assumed it to be constant, for simplicity. However, this study can be extended by modelling the flow rate or fluid velocity in the future.

\section{Acknowledgements}

This research was supported by the IVHM Centre, Cranfield University, UK and its industrial partners.

\section{References}

[1] Cheremisinoff, N. P. (1998), Liquid Filtration, Second Edition ed, Elsevier Inc.

[2] Sparks, T. (2011), Solid-Liquid Filtration: A User's Guide to Minimizing Cost \& Environmental Impact, Maximizing Quality \& Productivity, First Edition ed, Elsevier Science \& Technology Books.

[3] Sutherland, K. (2010), "Mechanical engineering: The role of filtration in the machinery manufacturing industry", Filtration and Separation, vol. 47, no. 3, pp. 24-27.

[4] Wilfong, D., Dallas, A., Yang, C., Johnson, P., Viswanathan, K., Madsen, M., Tucker, B. and Hacker, J. (2010), "Emerging challenges of fuel filtration", Filtration, vol. 10, no. 2, pp. 107-117.

[5] Jones, M., ( 2008), Engine Fuel Filter Contamination, QTR_03 ed., Boeing AeroMagazine.

[6] Ni, L. A., Yu, A. B., Lu, G. Q. and Howes, T. (2006), "Simulation of the cake formation and growth in cake filtration", Minerals Engineering, vol. 19, no. 10, pp. 1084-1097.

[7] Tien, C., Teoh, S. K. and Tan, R. B. H. (2001), "Cake filtration analysis - the effect of the relationship between the pore liquid pressure and the cake compressive stress", Chemical Engineering Science, vol. 56, no. 18, pp. 5361-5369.

[8] Tien, C. and Bai, R. (2003), "An assessment of the conventional cake filtration theory", Chemical Engineering Science, vol. 58 , no. 7 , pp. 1323-1336. 
[9] Endo, Y., Chen, D. -. and Pui, D. Y. H. (1998), "Effects of particle polydispersity and shape factor during dust cake loading on air filters", Powder Technology, vol. 98, no. 3, pp. 241-249.

[10] Abboud, N. M. and Corapcioglu, M. Y. (1993), "Modeling of Compressible Cake Filtration", Journal of colloid and interface science, vol. 160, no. 2, pp. 304-316.

[11] Tien, C. and Ramarao, B. V. (2013), "Can filter cake porosity be estimated based on the Kozeny-Carman equation?", Powder Technology, vol. 237, pp. 233-240.

[12] Wakeman, R. (2007), "Filter media: Testing for liquid filtration", Filtration and Separation, vol. 44, no. 3, pp. $32-34$.

[13] Carman, P. G. (1997), "Fluid flow through granular beds", Chemical Engineering Research and Design, vol. 75, no. 1 SUPPL., pp. S32-S46.

[14] Ergun, S. (1952), "Fluid Flow through Packed Columns", Chemical Engineering and Processing, vol. 48, pp. 89-94.

[15] Hamachi, M. and Mietton-Peuchot, M. (2001), "Cake thickness measurement with an optical laser sensor", Chemical Engineering Research and Design, vol. 79, no. 2, pp. 151-155.

[16] Liu, J., Swanson, J. J., Kittelson, D. B., Pui, D. Y. H. and Wang, J. (2013), "Microstructural and loading characteristics of diesel aggregate cakes", Powder Technology, vol. 241, pp. 244-251.

[17] Park, M. (2002), "Engine failure caused by erosion-corrosion of fuel manifold", Engineering Failure Analysis, vol. 9, no. 6, pp. 673-681.

[18] Niculita, O., Irving, P. and Jennions, I. K. (2012), "Use of COTS Functional Analysis Software as an IVHM Design Tool for Detection and Isolation of UAV Fuel System Faults", Annual Conference of the Prognostics and Health Management Society 2012, Vol. 3, Sep 22- 27, Minneapolis, USA, pp. Paper \#105.

[19] Niculita, O., Jennions, I. K. and Irving, P. (2013), "Design for diagnostics and prognostics: A physical-functional approach", Aerospace Conference, 2013 IEEE, pp. 1.

[20] Roussel, N., Nguyen, T. L. H. and Coussot, P. (2007), "General probabilistic approach to the filtration process", Physical Review Letters, vol. 98, no. 11.

[21] Sappok, A., Rodriguez, R. and Wong, V. (2010), "Characteristics and effects of lubricant additive chemistry on ash properties impacting diesel particulate filter service life", SAE International Journal of Fuels and Lubricants, vol. 3, no. 1 , pp. 705-722.

[22] Pontikakis, G. N., Koltsakis, G. C. and Stamatelos, A. M. (2001), "Dynamic filtration modeling in foam filters for diesel exhaust", Chemical Engineering Communications, vol. 188, pp. 21-46.

[23] Roychoudhury, I., Hafiychuk, V. and Goebel, K. (2013), "Model-based diagnosis and prognosis of a water recycling system", Aerospace Conference, 2013 IEEE, pp. 1.

[24] Baraldi, P., Di Maio, F., Mangili, F. and Zio, E. (2013), "A belief function theory method for prognostics in clogging filters", Chemical Engineering Transactions, vol. 33, pp. 847-852.

[25] Baraldi, P., Mangili, F. and Zio, E. (2015), "A prognostics approach to nuclear component degradation modeling based on Gaussian Process Regression", Progress in Nuclear Energy, vol. 78, no. 0, pp. 141-154.

[26] Saarela, O., Hulsund, J. E., Taipale, A. and Hegle, M. (2014), "Remaining Useful Life Estimation for Air Filters at a Nuclear Power Plant", 2nd internatial conference of the Prognostics and Health Management Society, . 
[27] Chen, Z. (2003), "Bayesian filtering: From Kalman filters to particle filters, and beyond", Statistics, vol. 182, no. 1, pp. 1-69.

[28] Zio, E. and Peloni, G. (2011), "Particle filtering prognostic estimation of the remaining useful life of nonlinear components", Reliability Engineering \& System Safety, vol. 96, no. 3, pp. 403-409.

[29] An, D., Choi, J. and Kim, N. H. (2013), "Prognostics 101: A tutorial for particle filter-based prognostics algorithm using Matlab", Reliability Engineering \& System Safety, vol. 115, no. 0, pp. 161-169.

[30] Baraldi, P., Compare, M., Sauco, S. and Zio, E. (2012), "Fatigue Crack Growth Prognostics by Particle Filtering and Ensemble Neural Networks", 1st european conference of the prognostics and health management society 2012, Vol. 3, 2012, Dresden, Germany, PHM Society, Dresden, Germany, .

[31] Cadini, F., Zio, E. and Avram, D. (2009), "Monte Carlo-based filtering for fatigue crack growth estimation", Probabilistic Engineering Mechanics, vol. 24, no. 3, pp. 367-373.

[32] Bechhoefer, E. (2008), "A method for generalized prognostics of a component using Paris law", ANNUAL FORUM PROCEEDINGS-AMERICAN HELICOPTER SOCIETY, vol. 64, no. 2, pp. 1460.

[33] Orchard, M., Kacprzynski, G., Goebel, K., Saha, B. and Vachtsevanos, G. (2008), "Advances in uncertainty representation and management for particle filtering applied to prognostics", Prognostics and Health Management, 2008. PHM 2008. International Conference on, pp. 1.

[34] Abbas, M., Ferri, A. A., Orchard, M. E. and Vachtsevanos, G. J. (2007), "An Intelligent Diagnostic/Prognostic Framework for Automotive Electrical Systems", Intelligent Vehicles Symposium, 2007 IEEE, pp. 352.

[35] Weiming Xian, Bing Long, Min Li and HouJun Wang (2014), "Prognostics of Lithium-Ion Batteries Based on the Verhulst Model, Particle Swarm Optimization and Particle Filter", Instrumentation and Measurement, IEEE Transactions on, vol. 63, no. 1, pp. 2-17.

[36] Daigle, M. J. and Goebel, K. (2013), "Model-Based Prognostics With Concurrent Damage Progression Processes", Systems, Man, and Cybernetics: Systems, IEEE Transactions on, vol. 43, no. 3, pp. 535-546.

[37] Butler, S. and Ringwood, J. (2010), "Particle filters for remaining useful life estimation of abatement equipment used in semiconductor manufacturing", Control and Fault-Tolerant Systems (SysTol), 2010 Conference on, pp. 436.

[38] Daigle, M. and Goebel, K. (2010), "Model-based prognostics under limited sensing", IEEE Aerospace Conference Proceedings, .

[39] Samie, M., Perinpanayagam, S., Alghassi, A., Motlagh, A. and Kapetanios, E., ( 2014), Developing Prognostic Models Using Duality Principles for DC-to-DC Converters.

[40] Saha, B., Celaya, J. R., Wysocki, P. F. and Goebel, K. F. (2009), "Towards prognostics for electronics components", Aerospace conference, 2009 IEEE, pp. 1.

[41] Jouin, M., Gouriveau, R., Hissel, D., Péra, M. and Zerhouni, N. (2014), "Prognostics of PEM fuel cell in a particle filtering framework", International Journal of Hydrogen Energy, vol. 39, no. 1, pp. 481-494.

[42] Fan, J., Yung, K. and Pecht, M. (2015), "Predicting long-term lumen maintenance life of LED light sources using a particle filter-based prognostic approach", Expert Systems with Applications, vol. 42, no. 5, pp. 2411-2420.

[43] Eker, O. F., Camci, F. and Jennions, I. K. (2013), "Filter Clogging Data Collection for Prognostics", Proceedings of the Annual Conference of the Prognostics and Health Management Society, 14-17 Oct 2013, New Orleans LA, USA, pp. 624-632. 
[44] Eker, O. F., Camci, F. and Jennions, I. K. (2014), "Physics-based Degradation Modelling for Filter Clogging", 2nd European Conference of the Prognostics and Health Management Society, 2014, Nantes, France, PHM Society, Nantes, France, .

[45] Saxena, A., Celaya, J., Balaban, E., Goebel, K., Saha, B., Saha, S. and Schwabacher, M. (2008), "Metrics for evaluating performance of prognostic techniques", 2008 International Conference on Prognostics and Health Management, PHM 2008, .

[46] Saxena, A., Celaya, J., Saha, B., Saha, S. and Goebel, K. (2009), "Evaluating algorithm performance metrics tailored for prognostics", IEEE Aerospace Conference Proceedings, .

[47] Saxena, A., Celaya, J., Saha, B., Saha, S. and Goebel, K. (2010), "Evaluating prognostics performance for algorithms incorporating uncertainty estimates", IEEE Aerospace Conference Proceedings, .

[48] Saxena, A., Celaya, J., Saha, B., Saha, S. and Goebel, K. (2010), "Metrics for Offline Evaluation of Prognostic Performance", International Journal of Prognostics and Health Management, vol. 1, no. 001, pp. 20.

[49] Saxena, A., Celaya, J., Saha, B., Saha, S. and Goebel, K. (2009), "On applying the prognostic performance metrics", International Conference on Prognostics and Health Management (PHM), San Diego, CA, USA, . 
2015-12-29

\section{Physics-based prognostic modelling of filter clogging phenomena}

Jennions, lan $\mathrm{K}$.

Elsevier

Omer F. Eker, Fatih Camci, Ian K. Jennions, Physics-based prognostic modelling of filter clogging phenomena, Mechanical Systems and Signal Processing, Volume 75, 15 June 2016, Pages 395-412

http://dx.doi.org/10.1016/j.ymssp.2015.12.011

Downloaded from Cranfield Library Services E-Repository 